\title{
Elegantes Durchwursteln am Krankenbett
}

Die Frage nach der gerechten Verteilung der Ressourcen im Gesundheitswesen wird in den letzten Jahren in zunehmendem Mass diskutiert. War früher der Mangel an Behandlungsmöglichkeiten limitierender Faktor, so ist es heute die Finanzierung des steigenden Angebotes, das an volkswirtschaftliche Grenzen stosse.

\author{
Jürg Nadig* \\ * Präsident Schweizerische Gesell- \\ schaft für medizinische Onkologie
}

Wie hoch der Anteil der Gesundheitskosten am Bruttosozialprodukt sein dürfe, wird kontrovers diskutiert. Die veränderte Bevölkerungsstruktur mit steigendem Anteil alter Menschen zusammen mit neuen Möglichkeiten der modernen Medizin führen zu einer steigenden Nachfrage mit einer Ausweitung des Konsums medizinischer Leistungen. Dass diese wirtschaftliche Prosperität des Gesundheitswesens, das sich immer neue Märkte erschliesst, im Zeitalter der Globalisierung und des neu erwachten wirtschaftlichen Liberalismus nicht bei allen auf eitel Freude stösst, liegt vor allem daran, dass die Leistungen nicht vom Konsumenten direkt, sondern von einem Dritten finanziert werden.

Dieser Dritte ist einerseits das Zwangskollektiv der in der obligatorischen Krankenversicherung (Grundversicherung) zu einer Solidargemeinschaft zusammengeschlossenen in der Schweiz Niedergelassenen, andererseits die öffentliche Hand. Letztere ist auf zwei Wegen an der Finanzierung des Gesundheitswesens beteiligt: Zum einen werden die Defizite der öffentlichen Spitäler zu 50\% aus Steuergeldern finanziert (Spitalfinanzierung). Zum andern werden öffentliche Gelder zur Verbilligung der Krankenkassenprämien eingesetzt: Die Finanzierung der vom neuen Krankenversicherungsgesetz (KVG) für alle Bürger vorgeschriebenen Grundversicherung wird in der Schweiz, als einzigem Land in Europa, über eine vom Einkommen des Versicherten unabhängige Kopfprämie finanziert. Um diese ungleiche Lastenverteilung zu mildern, sieht das KVG eine Prämienverbilligung für wirtschaftlich Schwächere vor, womit die Subventionsempfänger, verglichen mit der früheren Regelung, auf einen Drittel gesenkt werden konnte.

Korrespondenz:

Dr. med. Jürg Nadig

Bannhaldenstrasse 7 CH-8180 Bülach

Tel. 0448532611

juerg.nadig@hin.ch

\section{Drei mögliche Szenarien}

Die stete Zunahme der Behandlungsmöglichkeiten führte zu einer Ausweitung der Pflichtleistungen in der Grundversicherung. Zusammen mit der sich ändernden Altersstruktur der Bevölkerung mit einem steigenden Anteil der über 65jährigen führt dies zu steigenden Kosten, ausser es gelinge, die Preise für die Leistungen zu senken, was nachteilige Folgen für die Qualität haben könnte. Geht diese Entwicklung weiter, gibt es drei mögliche Szenarien:

1. Das Zwangskollektiv der Grundversicherten entscheidet, Gesundheit und Wohlfahrt seien das höchste Gut, weshalb alle möglichen medizinischen Leistungen gemäss dem Wunsche des Patienten von der Grundversicherung übernommen werden müssten, da eine Einschränkung des Leistungskatalogs einem unzumutbareren Sozialabbau gleichkomme. Die Schweiz als zweitreichstes Land könne sich hohe Kosten für das Gesundheitswesen leisten.

2. In der zweiten Variante wird keine obere Grenze für die Finanzierung des Gesundheitswesens festgelegt. Die Pflichtleistungen werden aber gemäss vorgegebenen Kriterien ausgewählt und die Kosten durch Rationalisierungsmassnahmen gesenkt oder mindestens dem Verlauf des Bruttosozialproduktes angepasst. Eine medizinische Massnahme wird als Pflichtleistung anerkannt, wenn sie wirksam, wirtschaftlich und zweckmässig ist. Dieses Szenarium entspricht der vom Bundesamt für Gesundheitswesen (BAG) verfolgten Politik: Die Kommission für Grundsatzfragen im Gesundheitswesen stellte fest, dass in der Schweiz eine Rationierung nicht notwendig sei, die Zweiklassenmedizin existiere nicht. Die Kosten im Gesundheitswesen könnten durch Rationalisierung unter Kontrolle gehalten werden.

3. In der dritten Variante wird in der Grundversicherung nur noch ein reduzierter Leistungskatalog finanziert. Es wird also nur noch ein Teil der wirksamen, zweckmässigen und wirtschaftlichen Behandlungen durch die Grund- 
versicherung finanziert, während andere Behandlungsmethoden nicht angeboten werden. In diesem Fall stellt sich dann die Frage, nach welchen Kriterien die Auswahl der Pflichtleistungen zu erfolgen hat.

Die Entscheide des Arztes sollen dem Kranken nicht schaden, ihm sogar nützen, seine Autonomie respektieren und gerecht sein. Diese medizinethischen Grundprinzipien gelten aber nicht nur am Krankenbett oder in der Sprechstunde, sondern für alle Bereiche des Gesundheitswesens. So lässt sich begründen, dass eine implizite Rationierung bei beschränkten Mitteln unzulässig ist, weil dadurch gegen das Gebot der Verfahrensgerechtigkeit verstossen wird.

Welche Mittel dem Patienten in der Grundversicherung zur Verfügung stehen sollen, müsste deshalb im politischen Diskurs festgelegt werden, damit nachvollziehbar wird, wem was in welcher Situation zusteht, damit der Patient nicht der Willkür des Arztes am Krankenbett oder dem Krankenkassenfunktionär ausgeliefert ist. Für einzelne Ökonomen ist eine solche Sichtweise wenn nicht blauäugig, so zumindest weltfremd, besser: marktfremd. Deshalb propagieren sie, die Ärzte müssten sich eben in den nächsten Jahren elegant «durchwursteln».

Was aber bedeutet dies in der onkologischen Praxis, am Krankenbett? In der medizinischen Onkologie stehen heute neue und erfreulich wirksame Medikamente zur Verfügung, die bei- spielsweise bei metastasierenden Kolorektalkarzinomen zu einer Vervierfachung der Überlebenszeit geführt haben. Gleichzeitig steigen aber die Kosten für die in der Grundversicherung zugelassenen Medikamente pro Behandlungsmonat um mehr als das Hundertfache an. Mit diesen teuren Medikamenten belaufen sich heute in der medizinischen Onkologie die durchschnittlichen jährlichen Fallkosten auf Fr. 7 000.-, beim Grundversorger sind es lediglich Fr. 550.-.

\section{Auswirkungen in der Praxis}

Wie könnte nun in Zukunft das «elegante Durchwursteln» in der onkologischen Praxis aussehen? Wird der Onkologe beim Aufklärungsgespräch über die neuen Therapiemöglichkeiten das Hauptgewicht auf die seltenen, potentiell gefährlichen Nebenwirkungen legen, in der Hoffnung, der Kranke verzichte dann auf die Therapie, was sich in günstigen Fallkosten niederschlägt und die Prämienzahler schont, während der Arzt erleichtert in der Krankengeschichte festhalten kann, der Patient habe nach ausführlicher Information autonom auf die risikoreiche Therapie verzichtet? Oder legt der Onkologe bei der Patientenaufklärung besonderes Gewicht auf die nachgewiesene Lebensverlängerung, so dass der Patient, trotz möglicher Risiken, als autonomer, aufgeklärter Bürger sich für die teure Therapie entscheidet, während sich der Arzt über die steigende Performance seines Pharmaaktienpaketes freut?

\section{Luzerner Trendtage Gesundheit Machbarkeit - Finanzierbarkeit - Ethik}

\section{Tagungsprogramm}

Die 2. Luzerner Trendtage Gesundheit widmeten sich der hochspezialisierten Medizin, insbesondere auch der sich immer weiter öffnenden Schere zwischen Machbarkeit und Finanzierbarkeit. Sie versuchten einen Beitrag zu leisten, die absehbaren Entwicklungen interdisziplinär und offen zu diskutieren. Am Beispiel der Rehabilitation und der Onkologie wurde zudem aufgezeigt, welche Auswirkungen die Spitzenmedizin auf andere Disziplinen hat, und dass auch in weniger spektakulären Fachbereichen Spitzenleistungen erbracht werden. Die jährlichen Tagungen stehen jeweils unter dem Motto «Machbarkeit - Finanzierbarkeit - Ethik».

\section{Veranstalter}

Träger der Veranstaltung ist das im Frühjahr 2004 gegründete Forum Gesundheit Luzern. Es steht unter dem Vorsitz des Luzerner Regierungsrats Dr. Markus Dürr. Es steht all jenen interessierten Kreisen zur Mitgliedschaft offen, die die Ziele des Vereins unterstützen.

\section{Kontakt und Information}

Forum Gesundheit Luzern, Horwerstrasse 87, 6005 Luzern,

Tel. 04131837 97, Fax 04131837 10,

E-Mail: info@trendtage-gesundheit.ch, Internet: www.trendtage-gesundheit.ch

Die Tagung vom nächsten Jahr findet am Mittwoch, 21., und Donnerstag, 22. März 2007, statt. 
Sollten wir dazu neigen, Entscheide im Interesse des Patienten gemäss den Prinzipien der Medizinethik zu fällen, statt uns elegant «durchzuwursteln», stellt sich die Frage, ob denn heute für eine Rationierung im Gesundheitswesen rationale Grundlagen vorliegen, um solche Zuteilungen nachvollziehbar durchzuführen? Die heute für die Registrierung von Medikamenten eingereichten Unterlagen beantworten diese Fragen nicht. Der klinischen Forschung in der Schweiz fehlen aber die finanziellen Mittel, solche klinisch wichtigen Fragen zu beantworten. Somit fehlen heute die notwendigen Entscheidungsgrundlagen für eine Rationierung.

Da die Erarbeitung von Rationierungsgrundsätzen und die Beschaffung von Entscheidungsgrundlagen für eine allfällige Rationierung Zeit benötigen, sollten wir bereits heute, noch ohne unmittelbaren Druck durch eine Notlage, diese Fragen in einer breiten Öffentlichkeit diskutieren und die notwendigen Entscheidungsgrundlagen erarbeiten. Da es nicht nur an politisch anerkannten Grundsätzen und Grundlagen für solch schwierige Entscheide mangelt, sondern auch die Kultur fehlt, solche Fragen anzugehen, diskutierte des Forum Gesundheit Luzern am 23. und 24. März 2006 an den 2. Luzerner Trendtagen Gesundheit unter dem Aspekt von Machbarkeit, Finanzierbarkeit und Ethik die Herausforderung durch die hochspezialisierte Medizin am Beispiel der Rehabilitation und der Onkologie. 